Vietnam Journal of Mechanics, VAST, Vol.28, No. 3 (2006), pp. 145- 154

\title{
ON THE COMPRESSION SOFTENING EFFECT OF PRESTRESSED BEAMS
}

\author{
Nguyen Van Khang, Nguyen Phong Dien, Nguyen Thi Van Huong \\ Department of Applied Mechanics, Hanoi University of Technology
}

\begin{abstract}
The main objective of the present paper is to study the transverse vibration of the prestressed beams. The differential equation of the transverse vibration of the Euler-Bernoulli beam is developed, in which the initial axial strain in every cross section of the beam is taken into account, so that the initial normal stress is not equal to zero. We have proposed some formulae to determine the natural frequencies of the prestressed beam. The forced transverse vibration of the beam with a moving external force has been considered. From this it follows compression softening effect of prestressed beams. A detailed comparison between the calculating results for the prestressed and the non-prestressed beam is also presented.
\end{abstract}

\section{INTRODUCTION}

In recent years, the prestressed ferro-concrete beams are usually used in branches of the construction and the road traffic. However, the research on the transverse vibration of the prestressed beams is still not extensively considered in Vietnam, although it is very necessary. The calculation of the stress of these beams is only confined to the evaluation of static stresses using the experimental formulae. The international literature on this respect is also little and a systematic study has not been shown. In some publications [1-8], the influence of axial forces or fatigue cracks on the natural frequencies of the beam, which is pre-compressed by the axial forces, was investigated. In the last case, the axial forces are usually supposed as constants.

The main objective of the present paper is to study in detail the transverse vibration of the prestressed beams. Firstly, the differential equation of the transverse vibration of the Euler-Bernoulli beam is established, in which the initial axial strain in every cross section of the beam is taken into account, so that the initial normal stress is not equal to zero. In the next sections, we propose some formulae to determine the natural frequencies of the prestressed beam. The forced transverse vibration of the beam with a moving external force has been considered. A comparison between the calculating results for the prestressed and the non-prestressed beam is also presented.

\section{DIFFERENTIAL EQUATION OF THE TRANSVERSE VIBRATION OF THE PRESTRESSED BEAM}

According to the Bernoulli beam theory, every cross section of the beam is always flat and during deformation perpendicular to the neutral axis. Note that the geometrical axis of the beam without deformation is a segment of a straight line. We choose this straight line as $x$-axis as shown in Fig.1. Neglecting the longitudinal and torsional oscillations, we consider only the transverse vibration of the beam in the direction of $z$-axis. 
In order to determine the differential equation for the transverse vibration of beams, we consider an infinitesimal volume as shown in Fig. 2. The length of this infinitesimal volume is assumed to be $d x$ and the mass is $d m$. Let $Q$ and $M_{y}$ be, respectively, the shear force and the bending moment acting in $x-z$ plane, and let $p(x, t)$ be the loading per unit length of the beam.

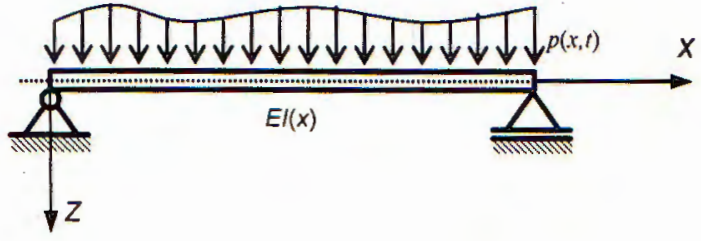

Fig. 1. Transverse vibration of a beam

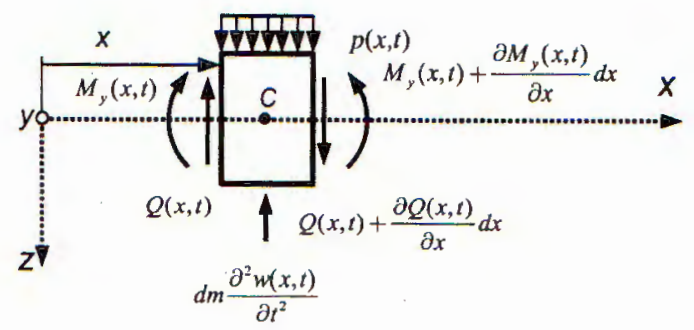

Fig. 2. Bending moments and shear forces

Neglecting the rotary inertia, the dynamic equilibrium conditions for the transverse vibration of bearns are obtained by applying D'Alembert principle [9] as

$$
\begin{gathered}
\sum F_{k z}=-d m \frac{\partial^{2} w}{\partial t^{2}}-Q+Q+\frac{\partial Q}{\partial x} d x+p(x, t) d x=0 \\
\sum m_{C}\left(\vec{F}_{k}\right)=-M_{y}+M_{y}+\frac{\partial M_{y}}{\partial x} d x-Q \frac{d x}{2}-\left(Q+\frac{\partial Q}{\partial x} d x\right) \frac{d x}{2}=0
\end{gathered}
$$

where $w(x, t)$ denotes the deflection of the beam. Equation (2.1) can be rewritten after simplification as

$$
\rho A(x) \frac{\partial^{2} w}{\partial t^{2}}=\frac{\partial Q}{\partial x}+p(x, t),
$$

where $\rho$ is the mass density and $A(x)$ denotes the cross-sectional area of the beam. From equation (2.2) we get

$$
Q=\frac{\partial M_{y}}{\partial x}
$$

From linear elastic theory, the bending moment $M_{y}$ and the normal stress $\sigma_{x x}$ in the direction of $x$-axis are given by

$$
M_{y}=\int_{A} z \sigma_{x x} d A, \quad \sigma_{x x}=E \varepsilon_{x x},
$$

where $\varepsilon_{x x}$ is the strain in $x$-axis. Assuming that the beam is prestressed, the initial strain in the cross section $A(x)$ must be taken in consideration, that is, $\varepsilon_{0}(x, z, 0)=\varepsilon_{0}(x)$. In the linear deformation region, strain $\varepsilon_{x x}$ is given in terms of the initial strain $\varepsilon_{0}$ as

$$
\varepsilon_{x x}(x, z, t)=\varepsilon_{0}(x)+\varepsilon_{x x}^{*}(x, z, t),
$$

where $\varepsilon_{x x}^{*}(x, z, t)$ is the strain caused by deformation of the beam. Fig. 3 shows an enlarged imagine of a part from the bended beam. The strain in a particular layer $z$ of the beam depends on coordinate $z$. If $z=z_{0}$, strain $\varepsilon\left(z_{0}\right)$ is equal to zero.

If the beam has a constant mass density and symmetric cross sections, the location of layer $z_{0}$ can be easily determined (it coincides commonly with the symmetric axis of the beam). However, in other cases, for example ferro-concrete beams, the neutral layer is not 
coincident with the symmetric axis of the beam. The length of the neutral layer between cross sections 1 and 2 can be determined from Fig. 3 as follows

$$
L_{0}=\rho_{z} d \varphi,
$$

where $\rho_{z}$ is the radius of curvature of the neutral layer. So the length of the layer $z$ between cross sections 1 and 2 before deformation is also equal to $L_{0}$. After deformation, this length becomes

$$
L=\left(\rho_{z}+z-z_{0}\right) d \varphi .
$$

Thus, the strain of the layer $z$ is

$$
\varepsilon_{x x}^{*}(z)=\frac{L-L_{0}}{L}=\frac{z-z_{0}}{\rho_{z}} .
$$

If we choose $z_{0}=0$, then Eq. (2.9) leads to

$$
\varepsilon_{x x}^{*}(z)=\frac{z}{\rho_{z}} .
$$

According to mathematical handbooks, the radius of curvature $\rho_{z}$ is given by

$$
\frac{1}{\rho_{z}}=-\frac{\frac{\partial^{2} w}{\partial x^{2}}}{\left[1+\left(\frac{\partial w}{\partial x}\right)^{2}\right]^{3 / 2}}
$$

Substituting Eq. (2.11) into Eq. (2.10) yields

$$
\varepsilon_{x x}^{*}(x, z, t)=-\frac{\frac{\partial^{2} w}{\partial x^{2}}}{\left[1+\left(\frac{\partial w}{\partial x}\right)^{2}\right]^{3 / 2}} z .
$$

Substituting Eq. (2.12) into Eq. (2.6), it shows that the strain of the prestressed beam is given by

$$
\varepsilon_{x x}(x, z, t)=\varepsilon_{0}(x)-\frac{\frac{\partial^{2} w}{\partial x^{2}}}{\left[1+\left(\frac{\partial w}{\partial x}\right)^{2}\right]^{3 / 2}} z \approx \varepsilon_{0}(x)-\frac{\partial^{2} w}{\partial x^{2}} z .
$$

Substituting Eq. (2.13) into Eq. (2.5) yields

$$
M_{y}=E \varepsilon_{0}(x) \int_{A} z d A-E \frac{\partial^{2} w}{\partial x^{2}} \int_{A} z^{2} d A .
$$

Note that

$$
\int_{A} z d A=w A(x), \quad \int_{A} z^{2} d A=I(x),
$$


where $I(x)$ denotes the moment of inertia of the cross section about the $y$-axis of the beam cross section. Eq. (2.14) takes the following form

$$
M_{y}=E A(x) \varepsilon_{0}(x) w-E I(x) \frac{\partial^{2} w}{\partial x^{2}} .
$$

Substituting Eq. (2.16) into Eq. (2.4) we obtain

$$
Q=\frac{\partial}{\partial x}\left[E A(x) \varepsilon_{0}(x) w\right]-\frac{\partial}{\partial x}\left[E I(x) \frac{\partial^{2} w}{\partial x^{2}}\right] .
$$

By substituting Eq. (2.17) into Eq. (2.3), we get a partial differential equation that governs the forced transverse vibration of the prestressed beam as follows

$$
\frac{\partial^{2}}{\partial x^{2}}\left[E I(x) \frac{\partial^{2} w}{\partial x^{2}}\right]-\frac{\partial^{2}}{\partial x^{2}}\left[E A(x) \varepsilon_{0}(x) w\right]+\rho A(x) \frac{\partial^{2} w}{\partial t^{2}}=p(x, t) .
$$

\section{FREE TRANSVERSE VIBRATION OF THE PRESTRESSED BEAM}

In the case that mass density and cross-sectional area of the beam are constant and the initial strains in every cross-sections are equal, the differential equation that describes the free transverse vibration of the prestressed beam can then be written as follows

$$
E I \frac{\partial^{4} w}{\partial x^{4}}-\varepsilon_{0} E A \frac{\partial^{2} w}{\partial x^{2}}+\rho A \frac{\partial^{2} w}{\partial t^{2}}=0 .
$$

Using Bernoulli method of the separation of variables, we assume a solution of Eq. (3.1) in the form

$$
w(x, t)=X(x) T(t)
$$

The functions $X(x)$ and $T(t)$ can be determined by the following equations [12]

$$
\begin{gathered}
\ddot{T}(t)+\omega^{2} T(t)=0 \\
X^{(I V)}(x)-\varepsilon_{0} \frac{A}{I} X^{\prime \prime}(x)-\frac{\omega^{2} \mu}{E I} X(x)=0
\end{gathered}
$$

where $\mu=\rho A$. The general solution of $\mathrm{Eq}$. (3.3) can be expressed in the form

$$
T(t)=D_{1} \cos \omega t+D_{2} \sin \omega t
$$

where the constants $D_{1}, D_{2}$ can be determined by using the initial conditions. By introducing the notations

$$
\alpha^{2}=\frac{\omega^{2} \mu}{E I}, \quad 2 \beta=\varepsilon_{0} \frac{A}{I}
$$

Eq. (3.4) can then be rewritten as

$$
X^{(I V)}(x)-2 \beta X^{\prime \prime}(x)-\alpha^{2} X(x)=0 .
$$

Eq. (3.7) is a fourth-order homogeneous linear differential equation with constant coefficients. The essential difference between the prestressed and the non-prestressed beam may be identified by the second term of Eq. (3.7). In the case of the non-prestressed beam $\varepsilon_{0}=0, \mathrm{Eq}$. (3.7) leads to the well-known equation $[9]$

$$
X^{(I V)}(x)-\alpha^{2} X(x)=0 .
$$

The characteristic equation of $\mathrm{Eq} .(3.7)$ is

$$
\lambda^{4}-2 \beta \lambda^{2}-\alpha^{2}=0
$$


The roots of this equation are given by

$$
\lambda^{2}=\beta \pm \sqrt{\beta^{2}+\alpha^{2}} .
$$

By introducing the notations

$$
\gamma(\alpha)=\sqrt{\sqrt{\beta^{2}+\alpha^{2}}-\beta}, \quad \delta(\alpha)=\sqrt{\sqrt{\beta^{2}+\alpha^{2}}+\beta},
$$

we obtain the solution from Eq. (3.10) as

$$
\begin{gathered}
\lambda_{1,2}= \pm i \sqrt{\sqrt{\beta^{2}+\alpha^{2}}-\beta}= \pm i \gamma, \\
\lambda_{3,4}= \pm \sqrt{\sqrt{\beta^{2}+\alpha^{2}}+\beta}= \pm \delta
\end{gathered}
$$

According to the theory on linear differential equations, the general solution of Eq. (3.7) is expressed in the form

$$
X(x)=C_{1} \cos \gamma x+C_{2} \sin \gamma x+C_{3} \cosh \delta x+C_{4} \sinh \delta x,
$$

where the constants $C_{1}, C_{2}, C_{3}$ and $C_{4}$ can be determined by using the boundary conditions. From Eq. (3.14) we get the following derivatives

$$
\begin{aligned}
& X^{\prime}(x)=-C_{1} \gamma \sin \gamma x+C_{2} \gamma \cos \gamma x+C_{3} \delta \sinh \delta x+C_{4} \delta \cosh \delta x \\
& X^{\prime \prime}(x)=-C_{1} \gamma^{2} \cos \gamma x-C_{2} \gamma^{2} \sin \gamma x+C_{3} \delta^{2} \cosh \delta x+C_{4} \delta^{2} \sinh \delta x \\
& X^{\prime \prime \prime}(x)=C_{1} \gamma^{3} \sin \gamma x-C_{2} \gamma^{3} \cos \gamma x+C_{3} \delta^{3} \sinh \delta x+C_{4} \delta^{3} \cosh \delta x
\end{aligned}
$$

Note that

$$
\cos 0=1, \quad \sin 0=0, \quad \cosh 0=1, \quad \sinh 0=0 .
$$

We consider prestressed uniform beams with boundary configurations shown in Fig. 4 and Fig. 5 as illustrating examples for calculating the natural frequencies of the prestressed beam.

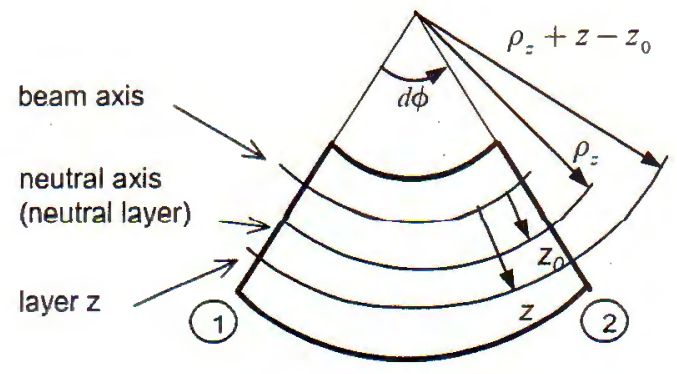

Fig. 3. Enlarged imagine of a part of the beam

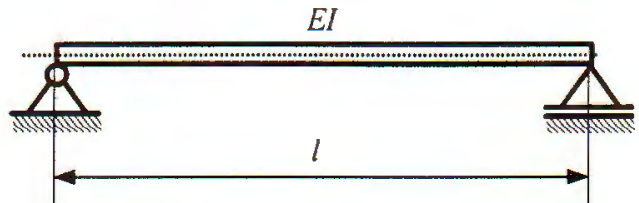

Fig. 4. The prestressed uniform beam with the hinged - hinged ends

In the first case (Fig. 4), the boundary conditions are

$$
X(0)=0, \quad X(l)=0, \quad X^{\prime \prime}(0)=0, \quad X^{\prime \prime}(l)=0 .
$$

Substituting these conditions into Eqs. (3.14) and (3.15) one yields the characteristic equation

$$
\sin \gamma l=0 .
$$



$[10]$

By using Eq. (3.18) we get the formula to determine natural frequencies of this beam

$$
\omega_{k}^{2}=k^{4} \frac{\pi^{4}}{l^{4}} \frac{E I}{\mu}+k^{2} \frac{\pi^{2}}{l^{2}} \varepsilon_{0} \frac{E}{\rho} \quad(k=1,2, \ldots) .
$$

For $k=1$, the fundamental natural frequency is

$$
\omega_{1}^{2}=\frac{\pi^{4}}{l^{4}} \frac{E I}{\mu}+\varepsilon_{0} \frac{\pi^{2}}{l^{2}} \frac{E}{\rho} .
$$

In the last case (Fig. 5), the boundary conditions are

$$
X(0)=0, \quad X(l)=0, \quad X^{\prime}(0)=0, \quad X^{\prime}(l)=0 .
$$

Similarly, we have the characteristic equation for the beam

$$
f(\alpha)=2 \beta \sin [\gamma(\alpha) l] \sinh [\delta(\alpha) l]+2 \alpha^{2}\{1-\cos [\gamma(\alpha) l] \cosh [\delta(\alpha) l]\}=0
$$

The roots $\alpha_{k}$ of Eq. (3.22) can be obtained by numerical calculation. The natural frequencies of the prestressed uniform beam with both clamped ends can then be expressed in terms of known values of $\alpha_{k}$ as

$$
\omega_{k}=\alpha_{k} \sqrt{\frac{E I}{\mu}}, \quad(k=1,2, \ldots) .
$$

The calculating results for this case are shown in Table 1 and Table 2.

Table 1. Results for a uniform beam with rectangular cross section $0.8(\mathrm{~m}) \times 0.7(\mathrm{~m}), l=8(\mathrm{~m})$, $E=0.3 \times 10^{11} \mathrm{~N} / \mathrm{m}^{2}$ and $\rho=2.3 \times 10^{3} \mathrm{~kg} / \mathrm{m}^{3}$

\begin{tabular}{|c|c|c|c|c|c|}
\hline$\varepsilon_{0}$ & $\omega_{1}$ & $\omega_{2}$ & $\omega_{3}$ & $\omega_{4}$ & $\omega_{5}$ \\
\hline-0.004 & 234.56 & 676.01 & 1349.11 & 2248.12 & 3372.71 \\
\hline-0.003 & 239.89 & 682.93 & 1356.56 & 2255.89 & 3380.68 \\
\hline-0.002 & 245.08 & 689.78 & 1363.98 & 2263.63 & 3388.63 \\
\hline-0.001 & 250.16 & 696.56 & 1371.35 & 2271.34 & 3396.56 \\
\hline 0.00 & 255.13 & 703.26 & 1378.68 & 2279.02 & 3404.47 \\
\hline 0.001 & 259.99 & 709.90 & 1385.97 & 2286.68 & 3412.36 \\
\hline 0.002 & 264.75 & 716.46 & 1393.22 & 2294.32 & 3420.24 \\
\hline 0.003 & 269.43 & 722.98 & 1400.43 & 2301.92 & 3428.09 \\
\hline 0.004 & 274.01 & 729.43 & 1407.61 & 2309.51 & 3435.93 \\
\hline
\end{tabular}

Based on these results, some comments can be made as follows

- In the case of the pre-compressed beam $\left(\varepsilon_{0}<0\right)$, the natural frequencies of the beam decrease for $\left|\varepsilon_{0}\right|$ increasing.

- In the case of the pre-strained beam $\left(\varepsilon_{0}>0\right)$, the natural frequencies of the beam increase with the raise of $\varepsilon_{0}$.

- The fundamental natural frequency of the beam is influenced significantly by the prestressed effect. However, this effect has fewer influence on high-order natural frequencies of the beam.

- The above-mentioned phenomenon is called "compression softening effect" of prestressed beams. 
Table 2. Results for a uniform beam with circular cross section, the radius is $0.45(\mathrm{~m})$, $l=10(\mathrm{~m}), E=0.3 \times 10^{11} \mathrm{~N} / \mathrm{m}^{2}$ and $\rho=2.3 \times 10^{3} \mathrm{~kg} / \mathrm{m}^{3}$

\begin{tabular}{|c|c|c|c|c|c|}
\hline$\varepsilon_{0}$ & $\omega_{1}$ & $\omega_{2}$ & $\omega_{3}$ & $\omega_{4}$ & $\omega_{5}$ \\
\hline-0.004 & 163.10 & 476.54 & 955.84 & 1596.26 & 3359.40 \\
\hline-0.003 & 168.00 & 482.82 & 962.56 & 1603.26 & 3366.70 \\
\hline-0.002 & 172.73 & 489.01 & 969.24 & 1610.23 & 3373.98 \\
\hline-0.001 & 177.33 & 495.12 & 975.88 & 1617.16 & 3381.24 \\
\hline 0.00 & 181.80 & 501.16 & 982.47 & 1624.07 & 3388.49 \\
\hline 0.001 & 186.16 & 507.11 & 989.01 & 1630.94 & 3395.72 \\
\hline 0.002 & 190.41 & 513.00 & 995.51 & 1637.79 & 3402.93 \\
\hline 0.003 & 194.55 & 518.80 & 1001.96 & 1644.61 & 3410.14 \\
\hline 0.004 & 198.60 & 524.54 & 1008.38 & 1651.40 & 3417.32 \\
\hline
\end{tabular}

\section{FORCED TRANSVERSE VIBRATION OF THE PRESTRESSED BEAM}

Using Eq. (2.18), we obtain the partial differential equation that describes the forced transverse vibration of the prestressed uniform beam with constant initial strain $\varepsilon_{0}$

$$
E I \frac{\partial^{4} w}{\partial x^{4}}-\varepsilon_{0} E A \frac{\partial^{2} w}{\partial x^{2}}+\rho A \frac{\partial^{2} w}{\partial t^{2}}=p(x, t) .
$$

Based on Bernoulli method, we assume a solution of Eq. (4.1) in the form

$$
w(x, t)=\sum_{k=1}^{\infty} X_{k}(x) q_{k}(t),
$$

where $X_{k}(x)$ is the eigenfunction, $q_{k}(t)$ is the unknown function that we have to find. By substituting Eq. (4.2) into Eq. (4.1), we obtain a system of ordinary differential equations to determine functions $q_{k}(t)$

$$
\ddot{q}_{k}(t)+\omega_{k}^{2} q_{k}(t)=\frac{\int_{0}^{l} p(x, t) X_{k}(x) d x}{\rho A \int_{0}^{l} X_{k}^{2}(x) d x}=h_{k}(t), \quad(k=1,2, \ldots) .
$$

We consider now the forced transverse vibration of a prestressed beam which is excited by a moving constant external force $F_{0}$ at constant velocity $v$ as shown in Fig. 6 .

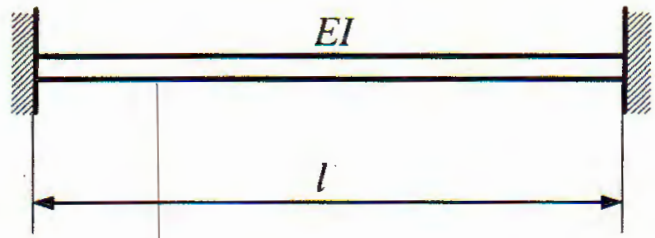

Fig. 5. The prestressed uniform beam with both fixed ends

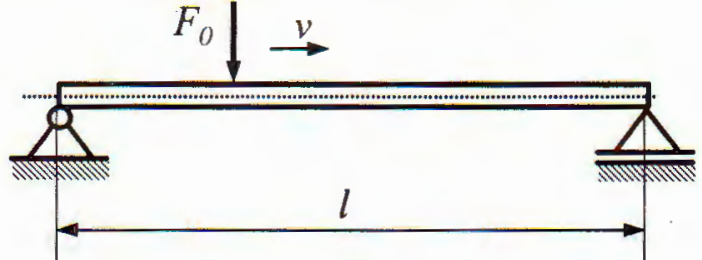

Fig. 6. Forced transverse vibration of a prestressed beam 
In this case, the eigenfunction $X_{k}(x)$ is chosen corresponding to the boundary configuration as $X_{k}(x)=\sin \frac{k \pi x}{l}$, so Eq. (4.3) can be rewritten in the following form

$$
\ddot{q}_{k}(t)+\omega_{k}^{2} q_{k}(t)=\frac{2 F_{0}}{\rho A l} \sin \Omega_{k} t, \quad(k=1,2, \ldots),
$$

where $\Omega_{k}$ is defined as

$$
\Omega_{k}=\frac{k \pi v}{l} .
$$

The general solution of Eq. (4.4) is given in the form

$$
q_{k}(t)=A_{k} \cos \omega_{k} t+B_{k} \sin \omega_{k} t+\frac{2 F_{0}}{\rho A l\left(\omega_{k}^{2}-\Omega_{k}^{2}\right)} \sin \Omega_{k} t .
$$

The constants $A_{k}, B_{k}$ can be determined using the initial conditions. Assuming that the initial conditions are

$$
\begin{aligned}
& w_{0}(x)=w(x, 0)=\sum_{k=1}^{\infty} X_{k}(x) q_{k} \\
& v_{0}(x)=\frac{\partial w(x, 0)}{\partial t} \doteq \sum_{k=1}^{\infty} X_{k}(x) \dot{q}_{k}
\end{aligned}
$$

Using the property of orthogonality of the eigenfunctions, these initial conditions can be simplified to

$$
q_{k}(0)=0, \dot{q}_{k}(0)=0, \quad(k=1,2, \ldots) .
$$

With the initial conditions (4.7), the constants $A_{k}, B_{k}$ are given by

$$
A_{k}=0, \quad B_{k}=-\frac{2 F_{0} \Omega_{k}}{\rho A l \omega_{k}\left(\omega_{k}^{2}-\Omega_{k}^{2}\right)} .
$$

Substituting Eq. (4.8) into Eq. (4.6) yields

$$
q_{k}(t)=-\frac{2 F_{0} \Omega_{k}}{\rho A l \omega_{k}\left(\omega_{k}^{2}-\Omega_{k}^{2}\right)} \sin \omega_{k} t+\frac{2 F_{0}}{\rho A l\left(\omega_{k}^{2}-\Omega_{k}^{2}\right)} \sin \Omega_{k} t .
$$

By substituting Eq. (4.9) and the eigenfunction $X_{k}(x)=\sin \frac{k \pi x}{l}$ into Eq. (4.2), we get the following formula for determining the dynamic deflection of the beam

$$
w(x, t)=\frac{2 F_{0}}{\rho A l} \sum_{k=1}^{\infty} \frac{1}{\omega_{k}^{2}-\Omega_{k}^{2}}\left[\sin \Omega_{k} t-\frac{\Omega_{k}}{\omega_{k}} \sin \omega_{k} t\right] \sin \frac{k \pi x}{l} .
$$

From Eq. (4.1), it can be shown that the resonant phenomenon occurs if $\Omega_{k}=\omega_{k}$. This leads to the following condition

$$
\frac{k^{2} \pi^{2}}{l^{2}} \sqrt{\frac{E I}{\rho A}+\varepsilon_{0} \frac{l^{2} E}{k^{2} \pi^{2} \rho}}=\frac{k \pi v_{k}^{*}}{l},
$$

where $v_{k}^{*}$ is the critical velocity which can be determined by using Eq. (4.11) as 


$$
v_{k}^{*}=\sqrt{\frac{k^{2} \pi^{2}}{l^{2}} \frac{E I}{\rho A}+\varepsilon_{0} \frac{E}{\rho}} .
$$

In the case that $v=v_{k}^{*}$ and $\Omega_{1}=\omega_{1}$ corresponding to $k=1$, for simplicity in writing we set $\Omega_{1}=\Omega, \omega_{1}=\omega, v_{1}^{*}=v^{*}$. The dynamic deflection of the beam for this case is given by

$$
w^{*}(x, t)=\frac{2 F_{0}}{\rho A l} \sin \frac{\pi x}{l} \lim _{\Omega \rightarrow \omega} \frac{\sin \Omega t-\frac{\Omega}{\omega} \sin \omega t}{\omega^{2}-\Omega^{2}} .
$$

By applying the L'Hospital law, we obtain

$$
w^{*}(x, t)=\frac{F_{0}}{\rho A l \omega^{2}} \sin \frac{\pi x}{l}(\sin \omega t-t \omega \cos \omega t) .
$$

Substituting $\omega=\Omega=\frac{\pi}{l} v^{*}$ into Eq. (4.14) yields

$$
w^{*}(x, t)=\frac{F_{0} l}{\rho A \pi^{2} v^{* 2}}\left[\sin \left(\frac{\pi v^{*}}{l} t\right)-\frac{\pi v^{*}}{l} t \cos \left(\frac{\pi v^{*}}{l} t\right)\right] \sin \frac{\pi x}{l} .
$$

The calculation of extrema of the function $w^{*}(x, t)$ leads

$$
w_{\max }^{*}(x)=\frac{F_{0} l^{3}}{\pi E\left(\pi^{2} I+\varepsilon_{0} l^{2} A\right)} \sin \frac{\pi x}{l} .
$$

The deflection in the middle of the beam (i.e. $x=\frac{l}{2}$ ) can then be calculated from Eq. (4.16) as

$$
w_{\max }^{*}\left(\frac{l}{2}\right)=\frac{F_{0} l^{3}}{\pi E\left(\pi^{2} I+\varepsilon_{0} l^{2} A\right)} .
$$

For $\varepsilon_{0}=0$ (i.e. in the case of the non-prestressed beam), Eq. (4.17) leads to the well-known formula [9]

$$
w_{\max }^{*}\left(\frac{l}{2}\right)=\frac{F_{0} l^{3}}{\pi^{3} E I} .
$$

A comparison between Eq. (4.17) and Eq. (4.18) gives us the following concluding remarks:

- The maximum deflection of the pre-compressed beam $\left(\varepsilon_{0}<0\right)$ is larger than the maximum deflection of the beam without prior compression.

- The maximum deflection of the pre-strained beam $\left(\varepsilon_{0}>0\right)$ is smaller than the maximum deflection of the beam without prior tension..

This behaviour illustrates the "compression softening effect" of prestressed beams which has been shown in the previous section.

\section{CONCLUSIONS}

In this article, the transverse vibration of prestressed beams is addressed. Some new results have been reached:

The partial differential equation that describes the transverse vibration of the prestressed beam is established generally by means of D'Alembert principle. This approach 
is more general and convenient than the method using a model of two compressive forces acting in both ends of the beam.

The calculating results for the problems of the free and forced transverse vibration of the prestressed beam have clearly demonstrated the "compression softening effect" which was found by means of experimental works in reference [5].

Based on the formulae developed in previous sections of the present article, it is possible to study complicated problems on dynamics of structures which contains prestressed elements. This will be the subject for future works.

Acknowledgment. This paper was completed with the financial support of the Vietnam Basic Research Program in Natural Science

\section{REFERENCES}

1. R. E. King and D. Rea, An Analysis of Damped Free Vibrations of Slender Prestressed Concrete Beams, Inter. Journal of Mech. Sci 7 (1965) 211-219.

2. A. D. Keer, On the Dynamic Response of Prestressed Beams, Journal of Sound and Vibration 49 (4) (1976) 569-573.

3. L. Tomski, J. Przybylski and T. Geister, Vibrations of a Prestressed Compound Beams with a Concentrated Mass, Journal of Sound and Vibration 174 (3) (1994) 315-321.

4. Y. L. Mo and W. L. Hwang, Dynamic Response of Prestressed Concrete Frames, Engineering Structures 16 (1994) 577-584.

5. M. Saiidi, B. Douglas and S. Feng, Prestress Force Effect on Vibration Frequency of Concrete Bridges, Journal of Structural Engineering 120 (7) (1994) 2233-2241.

6. S. Masoud, M. A. Jarran and M. Al-Maarory, Effect of Crack Depth on the Natural Frequency of a Prestressed Fixed-fixed Beam, Journal of Sound and Vibration 214 (2) (1998) 201-212.

7. E. Hamed and Y. Frostig, Free Vibrations of Cracked Prestressed Concrete Beams, Engineering Structures 26 (2004) 1611-1621.

8. R. Hinrichs, Gedämpfte Schwingungen vorgespannter Balken, Forschritt-Berichte VDI, Reihe 11, Nr. 142, VDI Verlag, Düsseldorf 1990.

9. Nguyen Van Khang, Engineering Vibration, fourth Edition (in Vietnamese), Science and Techniques Publishing House, Hanoi 2005.

10. Nguyen Thi Van Huong, On the Calculation of the Transverse Vibration of Prestressed Beams (in Vietnamese), Engineer-Thesis, Hanoi University of Technology 2005.

Received October 26, 2006

\section{VỀ HIỆU ỨNG LÀM MỀM DÂM BẰNG NÉN CƯA DẦM CÓ ỨNG SUẤT TRƯỚC}

Chủ đề chính của bài báo là nghiên cứu dao động uốn của dầm có ứng suất trước. Phương trình vi phân dao động uốn của dầm Euler-Bernoulli được thiết lập, trong đó ở mỗi mặt cắt ngang của dầm có biến dạng dài tỷ đối ban đầu, do đó ứng suất pháp ban đầu khác không. Trong bài báo này đã xây dựng một số công thức tính các tần số riêng của dầm có ứng suất trước và khảo sát dao động uốn cưỡng bức của dầm có ứng suất trước khi có ngoại lực di chuyển. Từ đó rút ra hiệu ứng làm mềm dầm bằng nén của dầm có ứng suất trước. Các kết quả tính toán cho dầm có ứng suất trước và cho dầm không có ứng suất trước đã được so sánh và đánh giá một cách chi tiết. 